\title{
Recent Advances and Future Opportunities with Magnetic Soft X-ray Microscopy
}

\author{
Peter Fischer ${ }^{1,2}$ \\ ${ }^{1}$ Materials Sciences Division, Lawrence Berkeley National Laboratory, Berkeley CA, USA \\ ${ }^{2}$ Physics Department, University of California, Santa Cruz, CA, USA
}

Nanomagnetism research aims to understand and control magnetic properties and behavior on the nanoscale through proximity and confinement. A wealth of information has been achieved in this reductionists approach, which to a large extent was made possible by the development of advanced instrumentation targeting the nanoscale. However, it has been recognized, that the next step beyond the nanoscale will be governed by mesoscale phenomena [1], since those are supposed to add complexity and functionality, which are essential parameters to meet future challenges specifically in magnetic technologies in terms of speed, size and energy efficiency of spin driven devices. Advances in development and application of multidimensional visualization techniques, such as tomographic magnetic imaging, and investigations of fast and ultrafast spin dynamics down to fundamental magnetic length and time scales with elemental sensitivity in emerging multi-component materials will be crucial to achieve mesoscience goals.

X-ray based spectro-microscopies offer a unique combination of both, diffraction limited spatial resolution in the nm regime, and temporal resolution across many scales dictated by the inherent time structures of x-ray sources, extending from fsec at X-ray lasers to the nsec regime at conventional storage rings. The polarization dependence of the $\mathrm{X}$-ray interaction with magnetic materials, which is manifested in x-ray circular dichroism effects, provides quantitative and inherently element- and chemical sensitivity allowing to map e.g. the local distribution of spin and orbital magnetic moments [2].

Magnetic soft X-ray microscopy can be performed in various ways. Among the real space imaging techniques are microscopies that use Fresnel zone plates as X-ray optical elements, such as magnetic full-field transmission soft X-ray microscopy (MTXM) and scanning transmission soft x-ray microscopy (STXM), as well as X-ray photoemission electron microscopies (X-PEEM). Those techniques provide routinely a spatial resolution at about $20 \mathrm{~nm}$ thus reaching out into fundamental magnetic length scales such as magnetic exchange lengths. MTXM covers in a single image a large field of view that allows investigating both the complexity, but also the stochasticity of magnetic processes, such as nucleation or reversal. Scanning transmission x-ray microscopy, although about 100x slower than a full-field TXM offers great flexibility in its photon detection schemes. Soft X-ray ptychography is an emerging X-ray technique, which has recently demonstrated about $10 \mathrm{~nm}$ spatial resolution for imaging magnetic domains [3].

Utilizing the inherent time structure of current synchrotron sources fast magnetization dynamics such as current induced wall and vortex dynamics in ferromagnetic elements can be performed with a stroboscopic pump-probe scheme with 70ps time resolution, limited by the lengths of the electron bunches [2].

In this talk I will review recent achievements and future opportunities with magnetic X-ray spectromicroscopies. Examples will include the static properties and dynamic behavior of magnetic vortex and skyrmion structures $[4,5,6]$ with potential application to novel magnetic logic elements $[7,8]$. We have 
found an asymmetry in the nucleation of magnetic vortex structures [4], that could be attributed to a DMI-like mechanism at the surface by micromagnetic simulations. Varying the exchange coupling between a PdCo disk with a strong perpendicular magnetic anisotropy and a conventional vortex structure in a permalloy disk we were able to imprint skyrmionic spin textures into the disks [9], where the interlayer thickness enable to tune the skyrmion number.

Time-resolved magnetic TXM studies in such disk structures provided a wealth of information ranging from control of circularity in the vortex [5] to an interlayer dependent gyrofrequency in the imprinted spin textures [10]. I will also present magnetic spectromicroscopy of domain walls, where we observe a decrease of the L3/L2 ratio, indicating a complex spin texture beyond the common Bloch or Neel type wall [11].

With magnetic soft x-ray ptychography in a thin SmCo5 film we have demonstrated recently the current capabilities in terms of spatial resolution and we have identified local inclusions, which we were able to analyze with magnetic spectromicroscopy[3].

Finally, I will show recent examples and approaches to image the 3dim magnetic domain structures in rolled-up thin films with x-ray tomography [12]. [13]

\section{References:}

[1] R. Service, Science 335, (2012) p. 1167

[2] P. Fischer, and H. Ohldag, Report on Progress in Physics 78 (2015) p 094501

[3] X. Shi et al, Applied Physics Letters (2016) accepted

[4] M.-Y.Im et al, Nature Communication 5 (2014) p. 5620

[5] V. Uhlír et al, Nature Nanotechnology 8 (2013) p 341-346

[6] S. Woo et al, G.S.D. Beach, Nature Materials (2016) accepted

[7] H. Jung et al, ACS Nano 6 (2012) p. 3712

[8] Z. Gu et al, Nature Communications 6 (2015) p. 6466

[9] R. Streubel et al, Scientific Reports 5 (2015) p 8787

[10] R. Streubel et al, Appl Phys Lett 107 (2015) p 112406

[11] M.J. Robertson et al, J Appl Phys 117 (2015) p. 17D145

[12] R. Streubel et al, Nature Communication 6 (2015) p. 7612

[13] This work was supported by the Director, Office of Science, Office of Basic Energy Sciences, Materials Sciences and Engineering Division, of the U.S. Department of Energy Contract No. DEAC02-05-CH1123 in the Non-Equilibrium Magnetic Materials Program. 